\title{
Emerging New Trends in Hybrid Vehicle Localization Systems
}

\author{
Nabil Drawil and Otman Basir \\ Department of Electrical and Computer Engineering, University of Waterloo \\ Canada
}

\section{Introduction}

Over the last decade, vehicle localization has been attracting attention in a wide range of applications. A number of localization techniques have been developed to serve a variety of applications Al-Bayari \& Sadoun (2005); Aono et al. (1998); Bouju et al. (2002); Cramer (1997); Dao et al. (2002); Drawil \& Basir (2010); Jabbour, Cherfaoui \& Bonnifait (2006); Lai \& Tsai (2003); Nishimura et al. (1996); Sliety (2007); Stockus et al. (2000). In recent years, the focus has been on localization accuracy improvement - an issue considered crucial, specially in mission critical applications. For instance, for emergency response systems, such as the eCall system, to deliver on their task they need reliable and accurate localization capabilities. These capabilities are becoming as important in other applications, including, accident avoidance and management, navigation systems, location sensitive billing systems, location based services.

The focus of much recent research in localization has been on improving accuracy through the use of multiple localization modalities. This chapter provides a review on multi-modality based localization techniques and establishes a categorization of such techniques based on the type of measurement and the strategy employed to fuse measurements from multiple localization sources.

Although these techniques have demonstrated significant performance improvement, there remain situations that give rise to degraded localization accuracy. Moreover, current localization systems lack in their ability to reliably quantify the accuracy of localization estimates, neither the means by which sources of localization information are properly discounted based on reliability/accuracy merits.

In this chapter, a novel framework is proposed to tackle the aforementioned issues. The proposed framework fuses different localization techniques in order to improve their location estimates, and provides a location reliability assessment that captures the integrity of the estimates. Knowledge about estimate integrity allows the system to plan the use of its localization resources so as to match the target accuracy of the application. The proposed framework provides the tools that would allow for modeling the impact of the operation conditions on estimate integrity, as such it enables more robust system performance.

\section{Motion and GPS measurement data fusion}

Differential GPS (DGPS) and Assisted GPS (A-GPS) are two advanced types of GPS technologies that provide a high level of accuracy and fast retrieving rate. Nevertheless, using 
a GPS receiver as the sole vehicle localization measurement source may turn to be unreliable, especially in urban canyons and other areas where the satellite signal can be distorted or lost. A number of solutions have been reported in the literature that proposed augmenting GPS measurements with information about the vehicle's motion in order to improve localization accuracy. In what follows we provide a summary of a number of such solutions.

\subsection{Dead Reckoning (DR) and GPS integration}

A DR is a localization method that estimates the next location of a mobile object over a series of short time intervals, given the object's direction, speed, and previous location. DR is simple and known for producing incremental error and hence needs to be reset periodically. It is therefore suitable for use over short periods of time.

One approach to resetting the accumulative localization error is to combine DR with GPS whereby GPS measurements are used to reduce the DR accumulative error; when the GPS measurement is unavailable, the DR estimates the location using sensors such as wheel odometers, a flux-gate compass, a gyroscope, and an accelerometer Kao (1991).

\subsection{Inertial Navigation System (INS) and GPS fusion}

Basically, INS operates as a DR system. INS employs a computing unit and motion sensors to estimate its location without relying on any external reference once it is initialized using for example a GPS measurement. To avoid the accumulated error caused by the measurements of internal sensors in INS, the INS location estimate is fused with measurement data from other sources. As discussed in Skog \& Handel (2009) fusing INS and GPS can take the form of a loosely or tightly coupled system architecture.

An example of a system that fuses INS and GPS is the real-time kinematic global positioning system (RTK GPS) Bouvet \& Garcia (2000) which uses an Extended Kalman Filter (EKF) to fuse data. In this system, GPS latency is defined as the time required for the satellite signals to travel to Earth and the time required for the computation of the location; GPS latency varies with the number of observed satellites. Therefore, the GPS latency is encapsulated in the EKF state so that the fusion of the INS and GPS data is synchronized with the readings of the sensors.

It is possible to fuse standard GPS and INS by means of a KF as well Honghui \& Moore (2002). In this case the computational complexity of the EKF can be reduced by preprocessing the INS measurements and inputting them into the $\mathrm{KF}$ as a linear component. However, preprocessing the INS measurement adds to the computational cost of the solution.

\subsection{Other motion sensors and DGPS fusion}

Integrating the INS of a dynamic model with a DGPS is also investigated inRezaei \& Sengupta (2005). To deal with the nonlinearity of the dynamic model, an EKF is used. Due to the accelerometer noise other motion sensors, such as six wheel-speed encoders, a steering angle encoder, and an optical yaw rate gyro, are used instead. Localization accuracy of $0.9 \mathrm{~m}$ on 100 $\mathrm{m}$ driving track was reported for situations where the system relies on the dynamic model more than it does on the GPS measurements. The multipath effect is not addressed as the experiment was conducted in an open space environment.

In Aono et al. (1998) a method of positioning a vehicle on undulating ground by fusing DGPS data and motion sensor data is proposed. A fibre optic gyro, a roll pitch sensor, and wheel 
encoders are used as motion sensors. The positioning accuracy is improved by compensating for the error for each sensor. The error is determined by means of a KF, which is also utilized as a fusion unit.

In Sharaf et al. (2005) an Artificial Neural Network (ANN) is chosen as a tool for detecting errors and noises in INS measurements using a DGPS as a guide to the true location of the vehicle during a training phase. The work reported in Sharaf et al. (2005) is similar to that reported in Bouvet \& Garcia (2000) in that preprocessing operations are performed on the measurements before they are fused. An assumption that is made in this method is that the DGPS data is always either available or unavailable due to an outage in satellite signal. However, in urban areas, satellite signals are often available but quite often are contaminated by multipath noises, which effects the quality of the ANN learning.

\section{Fusion of landmark, INS, and GPS measurements}

Detecting and recognizing landmarks provide spatial information related to the local environment. It is therefore possible to integrate spatial information with localization measurements from DR and GPS in order to improve localization accuracy Fuerstenberg \& Weiss (2005); Jabbour, Bonnifait \& Cherfaoui (2006); Jabbour, Cherfaoui \& Bonnifait (2006); Rae \& Basir (2007); Weiss et al. (2005). Two approaches for detecting and augmenting landmarks to vehicle localization systems are presented next along with another localization technique that attempts to detect visible satellites for use in the positioning process.

\subsection{Laser scanners, digital maps, and GPS/DR}

Due to the accumulated error caused by the long satellite outages in GPS/DR localization systems, digital maps are utilized to perform localization during such outages Weiss et al. (2005). A laser scanner mounted on a vehicle scans major objects in the vehicle environment. The system matches these landmarks with other landmarks in the digital map that represent the region of interest. If there is a match, the vehicle location is estimated by correlating the identified landmarks.

However, segmentation is not a trivial job specially in situations where landmarks are merged with background objects. Moreover, the system must be trained by having it traverse the regions of interest Fuerstenberg \& Weiss (2005) to extract landmarks (features, such as traffic signs and the posts of traffic lights) that can later be used as a reference points.

In Jabbour, Bonnifait \& Cherfaoui (2006), a vehicle equipped with an autonomous navigation system and a laser scanner is reported. The laser scanner is used to detect the edges of sidewalks and estimate the distance between the edge of the sidewalk and the vehicle. Distance measurements are utilized to improve the accuracy of a localization system that comprises GPS, DR, and Geographic Information System (GIS). The GIS data contains digitized information such as abstract road maps, road edges, and other landmarks. Landmark information is created through a learning stage. During the testing stage, the EKF fusion technique produces an innovation value from which the system determines whether to accept the fusion location estimate. If the GPS data is corrupted by multipath signals or is unavailable, only the DR location estimate utilized. The vehicle location estimate is used to select the region of interest from the GIS database that contains the landmark information. To improve the vehicle location estimate, a matching scheme is performed to compare the GIS-extracted landmarks (i.e., sidewalk edges) with those extracted by the laser scanner, and 
the estimated distances between the sidewalk edge and the vehicle are then used in fixing the vehicle location. Although the memory constraints are overcome by using the GIS, the accuracy of the estimate of the distances is not consistent due to occluding objects between the laser scanner and the edge of the sidewalk. The training phase required for any traversed region is also not insignificant.

\subsection{Vision, digital maps, and GPS/DR}

Visual data is also utilized in localization techniques since digital images can provide a wide range of information about the surrounding environment. Due to the time required for image processing Jabbour, Cherfaoui \& Bonnifait (2006), only key images are maintained and linked to the GIS database Jabbour, Bonnifait \& Cherfaoui (2006). Again, both GPS/DR are used and the proximity of the vehicle location estimate to the roads in the GIS database is examined. The road segment closest to the location estimate is then selected, and key images of that road are extracted in order to compare their features with the features of the images taken during the navigation stage. The weakness of this strategy appears when the curvature of the vehicle's path is significant, especially when the vehicle turns in orthogonal intersections.

Visual features can, however, be blended with other location measurements, such as GPS and DR data in the EKF formulation Rae \& Basir (2007). The main advantage of this strategy is that the uncertainty of all the information sources is kept local to the EKF, namely, in the error covariance matrix, which guarantees a minimum mean square error estimates. In Rae \& Basir (2007), the EKF structure is derived and validated where the curvature of the roads is employed as a visual feature. It is shown that when the roads are curvy, the vehicle location estimate is dramatically improved. On the other hand, if the road traversed is not curved, then the accuracy of the location estimate remains the same as that produced by the GPS/DR fusion localization technique.

\subsection{Satellite visibility and DGPS}

In urban areas, GPS multipath signals cause unpredictable localization errors due to the NLOS satellite signals. Another approach is the localization system which is driven by tracking visible GPS satellites using an infrared camera. An omni-directional infrared camera mounted on the top of a vehicle is used to recognize obstacles and their height and to detect visible satellites by observing their positions with a satellite orbit simulator Meguro et al. (2009). This method allows the system to exclude any radio waves emitted by invisible satellites to improve the localization accuracy.

The vehicle localization system used in this approach has high degree of accuracy since it employs a DGPS receiver. However, in high rise building areas, the availability of location estimates is low due to the lack of enough visible satellites, and even with enough visible satellites, the geometric configuration of the constellation may result in a high Dilution of Precision (DOP).

\section{Cooperative localization}

Cooperative Localization is a recent location estimation approach that has been implemented in vehicular positioning and wireless communication systems. This localization scheme is suitable for scenarios which involve the coexistence of several entities that independently provide location information. The goal is to localize a mobile node or to enhance its location 
estimate given that it shares relative spatial information with nearby nodes (e.g., other vehicles or mobile network towers).

\subsection{Radio signal measurement data fusion}

Radio localization methods have been studied extensively for cellular networks in a wide range of applications (e.g., for CDMA networks see Al-Jazzar \& Caffery (2004); Caffery \& Stuber (1994; 1998); Caffery (2000); Le et al. (2003); McGuire et al. (2003); Porretta et al. (2008); Sayed et al. (2005); Venkatraman et al. (2002); Wang et al. (2003); Wylie \& Holtzman (1996) and for GSM networks see Chen et al. (2006)). An example of these systems is a localization system that estimates the locations of emergency calls initiated by cellular phones. The system operates on the principle that measurements from different Base Stations (BS's) are combined in order to compute the location of a Mobile Station (MS). The BS's typically have different levels of uncertainty in their measurements, which are minimized as a result of the fusion process. The relative spatial information in this system is based on the measurements from radio signals, such as Time of Arrival (TOA), Time Difference of Arrival (TDOA), Angel of Arrival (AOA), Received Signal Strength (RSS). In some of these GPS-less approaches, a mix of two or more different types of radio signal measurements is utilized in order to relax constraints such as the synchronization of the BS's.

In the following subsections detailed models for some of these techniques are given. $\left(x_{m}, y_{m}\right)$ signifies the MS location. The locations of $n$ base stations: $\left(\mathrm{BS}_{1}, \mathrm{BS}_{2}, \mathrm{BS}_{3}, \ldots, \mathrm{BS}_{n}\right)$ are denoted by $\left\{\left(x_{1}, y_{1}\right),\left(x_{2}, y_{2}\right),\left(x_{3}, y_{3}\right), \ldots,\left(x_{n}, y_{n}\right)\right\}$, respectively. For simplicity and without loss of generality, locations are represented by two coordinates, $x$ and $y$, in the Cartesian coordinate system.

\subsubsection{TOA data fusion}

Time of arrival measurements are based on the time of flight of a signal as it travels between a source and a destination. Since the signal travels at the speed of light $(c)$, it is possible to compute the distance between the two points as follows:

$$
d_{i}=\left(t_{i}-t_{m}\right) c
$$

where $t_{m}$ signifies the signal sending time from the MS, $t_{i}$ signifies the signal arrival time at the $\mathrm{BS}_{i}$, and $i$ signifies the BS's index (i.e., $i=\{1,2, \ldots, n\}$ ).

According to Caffery \& Stuber (1998), the TOA technique can be employed using three BS's, the minimum number of reference points in two dimensions (Figure 1), in order to estimate the MS location by computing the distances between each BS and the MS (i.e., $d_{1}, d_{2}, d_{3}$ ), as per Equation 1, and then formulating the following optimization problem:

$$
\hat{x}_{m}, \hat{y}_{m}=\arg \min _{x_{m}, y_{m}} \sum_{i=1}^{3}\left(d_{i}-\sqrt{\left(x_{i}-x_{m}\right)^{2}+\left(y_{i}-y_{m}\right)^{2}}\right)^{2}
$$

Nevertheless, due to possible NLOS propagation conditions, the actual Euclidean distances between the MS and the $\mathrm{BS}_{i}$ is less than or equal to $\left(t_{i}-t_{m}\right) c$. This inequality creates more than one solution for the optimization problem in 2, all of which reside in a bounded area, as shown in Figure 1. A constrained version of the optimization problem in 2 is proposed in Caffery (1999); Porretta et al. (2008) in order to increase the localization accuracy; however, the 


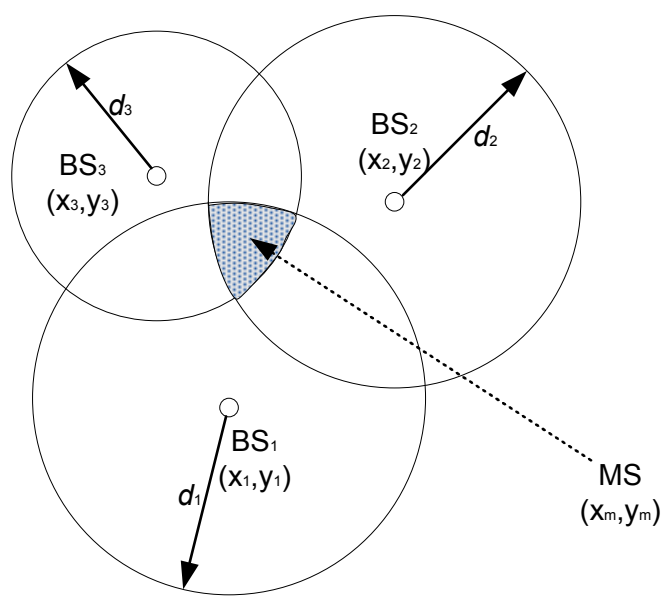

Fig. 1. The TOA localization method.

geometric arrangement of the BS's may produce a poor location estimates due to the shape of the bounded area that contains the MS. This shortcoming might be avoided using more BS's. The next method described below utilizes more than three BS's in estimating the MS location so that ambiguity in the distance computation is reduced.

In Caffery (2000); Sayed et al. (2005) the Cartesian coordinate system is represented as follows. The location of one of the base stations is assumed to be the origin (e.g., $\mathrm{BS}_{1}$ be the origin: $\left.\left(x_{1}, y_{1}\right)=(0,0)\right)$ and the locations of the other objects in the network are computed with respect to the origin. Hence, the distances $\left(d_{1}, d_{2}, d_{3} \ldots, d_{n}\right)$ can be used to estimate the location of the MS by solving the following set of equations:

$$
\begin{aligned}
& d_{1}^{2}=x_{m}^{2}+y_{m}^{2} \\
& d_{2}^{2}=\left(x_{2}-x_{m}\right)^{2}+\left(y_{2}-y_{m}\right)^{2} \\
& d_{3}^{2}=\left(x_{3}-x_{m}\right)^{2}+\left(y_{3}-y_{m}\right)^{2} \\
& \quad \vdots \\
& d_{n}^{2}=\left(x_{n}-x_{m}\right)^{2}+\left(y_{n}-y_{m}\right)^{2}
\end{aligned}
$$

After rearranging terms, the above equations can be written as follows:

$$
\left[\begin{array}{cc}
x_{2} & y_{2} \\
x_{3} & y_{3} \\
\vdots & \vdots \\
x_{n} & y_{n}
\end{array}\right]\left[\begin{array}{l}
x_{m} \\
y_{m}
\end{array}\right]=\frac{1}{2}\left[\begin{array}{c}
k_{2}^{2}-d_{2}^{2}+d_{1}^{2} \\
k_{3}^{2}-d_{3}^{2}+d_{1}^{2} \\
\vdots \\
k_{n}^{2}-d_{n}^{2}+d_{1}^{2}
\end{array}\right]
$$


where $k_{i}^{2}=x_{i}^{2}+y_{i}^{2}$. Equation 4 can be expressed in a matrix form

$$
\mathbf{H x}=\mathbf{b}
$$

where $\mathbf{H}=\left[\begin{array}{cc}x_{2} & y_{2} \\ x_{3} & y_{3} \\ \vdots & \vdots \\ x_{n} & y_{n}\end{array}\right], \mathbf{x}=\left[\begin{array}{l}x_{m} \\ y_{m}\end{array}\right]$, and $\mathbf{b}=\frac{1}{2}\left[\begin{array}{c}k_{2}^{2}-d_{2}^{2}+d_{1}^{2} \\ k_{3}^{2}-d_{3}^{2}+d_{1}^{2} \\ \vdots \\ k_{n}^{2}-d_{n}^{2}+d_{1}^{2}\end{array}\right]$.

Equation 5 represents an overdetermined system (i.e., $n>2$ ). Practically, such a system has no exact solution. Therefore a linear least squares method is used to estimate the location of the MS as follows:

$$
\hat{\mathbf{x}}=\left(\mathbf{H}^{T} \mathbf{H}\right)^{-1} \mathbf{H}^{T} \mathbf{b}
$$

where $(.)^{T}$ signifies matrix transpose and $(.)^{-1}$ signifies matrix inverse.

Alternative techniques, such as the maximum likelihood are reported in McGuire et al. (2003); Wang et al. (2003).

\subsubsection{TDOA data fusion}

TDOA is preferable to the TOA due to the fact that TDOA does not require synchronization between the MS and BS's, Figure 2. Instead, it takes advantage of the synchronization of the CDMA cellular network BS's to compute the difference between the time of arrivals of the MS

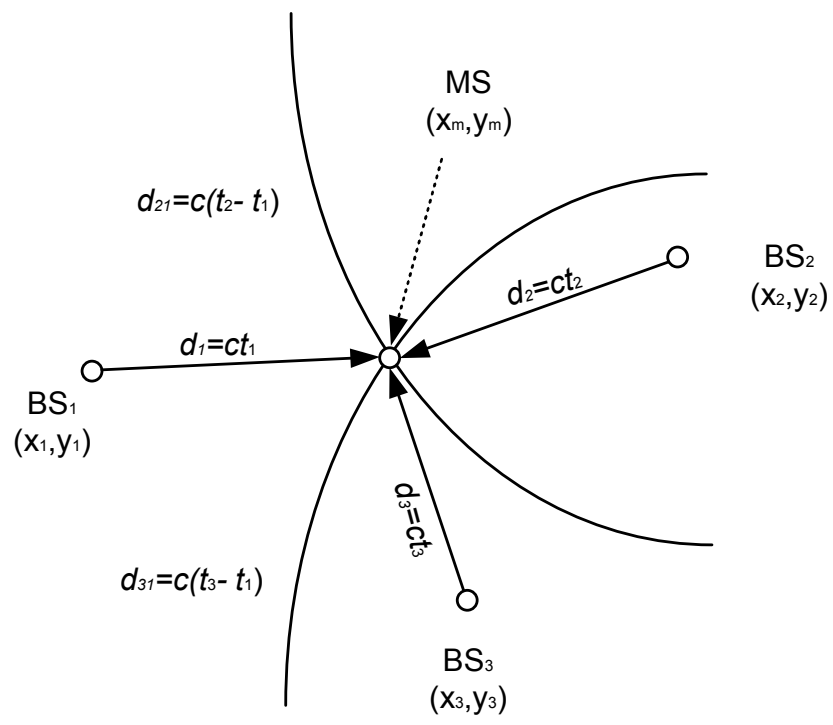

Fig. 2. The TDOA localization method. 
signal at the $\mathrm{BS}_{i}$ and $\mathrm{BS}_{1}$, where $i \in\{2,3, \ldots, n\}$. The difference in the distance is therefore defined as follows:

$$
\begin{aligned}
d_{i 1} & \equiv d_{i}-d_{1} \\
& =\left(t_{i}-t_{m}\right) c-\left(t_{1}-t_{m}\right) c \\
& =\left(t_{i}-t_{1}\right) c
\end{aligned}
$$

It can be seen that the difference is not affected by errors in the MS clock time $t_{m}$. Substituted Equation 7 in Equation 3, and then expanding and rearranging the terms produce the following set of equations:

$$
\left[\begin{array}{cc}
x_{2} & y_{2} \\
x_{3} & y_{3} \\
\vdots & \vdots \\
x_{n} & y_{n}
\end{array}\right]\left[\begin{array}{l}
x_{m} \\
y_{m}
\end{array}\right]=d_{1}\left[\begin{array}{c}
-d_{21} \\
-d_{31} \\
\vdots \\
-d_{n 1}
\end{array}\right]+\frac{1}{2}\left[\begin{array}{c}
k_{2}^{2}-d_{21}^{2} \\
k_{3}^{2}-d_{31}^{2} \\
\vdots \\
k_{n}^{2}-d_{n 1}^{2}
\end{array}\right]
$$

which can be expressed in a matrix form as follows:

$$
\mathbf{H x}=d_{1} \mathbf{c}+\mathbf{r}
$$

where $\mathbf{H}=\left[\begin{array}{cc}x_{2} & y_{2} \\ x_{3} & y_{3} \\ \vdots & \vdots \\ x_{n} & y_{n}\end{array}\right], \mathbf{c}=\left[\begin{array}{c}-d_{21} \\ -d_{31} \\ \vdots \\ -d_{n 1}\end{array}\right]$, and $\mathbf{r}=\frac{1}{2}\left[\begin{array}{c}k_{2}^{2}-d_{21}^{2} \\ k_{3}^{2}-d_{31}^{2} \\ \vdots \\ k_{n}^{2}-d_{n 1}^{2}\end{array}\right]$.

Similarly, Equation 9 can be solved using the following linear least squares formulation:

$$
\hat{\mathbf{x}}=\left(\mathbf{H}^{T} \mathbf{H}\right)^{-1} \mathbf{H}^{T}\left(d_{1} \mathbf{c}+\mathbf{r}\right)
$$

The solution of Equation 10 is determined in two steps. First, the estimate of the MS is determined in terms of $d_{1}$, which is substituted in the quadratic expression $d_{1}^{2}=x_{m}^{2}+y_{m}^{2}$ to compute $d_{1}$. Second, the value of $d_{1}$ is substituted back in Equation 10 to solve for $\hat{\mathbf{x}}$ Sayed et al. (2005).

\subsubsection{AOA data fusion}

AOA techniques estimate the location of an MS by measuring the angle of signal arrival from the MS at several BS's by means of an antenna array. The MS location is then estimated through the intersection of the straight paths leaving from at least two BS's, as depicted in Figure 3. However, combining only two AOA measurements may introduce a large amount of uncertainty with respect to the MS location estimate, especially when the MS is close to the line connecting the two BS's. Moreover, this localization method requires the MS to be in LOS with the participating BS's, since reflected or diffracted signals result in misleading information. For this reason, it is preferable for the AOA to be combined with another localization method, such as TOA or TDOA.

\subsubsection{RSS data fusion}

RSS based localization is a method that employs mathematical models that describe the path loss as a function of distance. Since these models translate the received signal power into a 


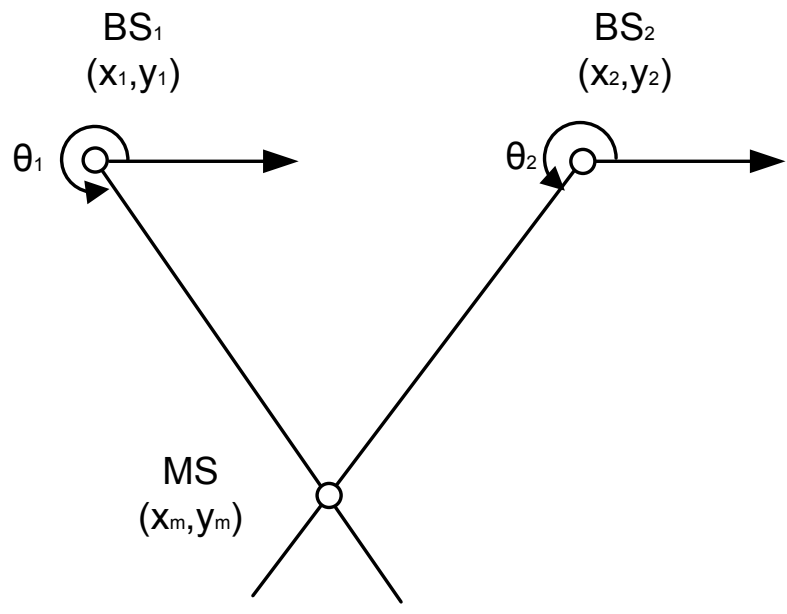

Fig. 3. The AOA localization method.

distance between an MS and a BS, the MS must lie on a circle centered at the BS. Employing three or more BS's provides an estimate for the MS location.

RSS is well known for being drastically affected by multipath fading and shadowing (multipath signals). The error caused by multipath signals can be reduced by using prior knowledge available on the contours of the signal strength centered at the BS's Smith (1991). However, such knowledge assumes a specific surrounding environment that can change due to change in whether, moving objects, such as trucks, as well as new buildings and other barriers.

\subsubsection{Fingerprinting}

This localization method is a pattern recognition, or pattern matching, technique. The underlying concept of fingerprinting is that the radio signal propagation characteristics of an MS are unique in terms of TOA, AOA, and RSS when captured at different BS's Chen et al. (2006); Porretta et al. (2008). These characteristics can therefore be used as a signature to indicate the location of an MS. The fingerprinting method has two phases: a training phase and localization phase. In the training phase, a database is created to index the different patterns in the characteristics of the radio signal propagation. In the localization phase, the signature of the MS is matched with the patterns in the database. The challenging aspect of this method is assuring that the system can distinguish between similar patterns that represent different locations.

Of course, the more exhaustive is the training phase (i.e., recording a signature for every small area in the environment), the more accurate is the MS location estimate. The main drawback of this method is the requirement to continually update the database as the configuration of 
the BS's changes when BS's are removed or new ones are added. Nevertheless, this method is becoming more attractive for indoor applications because the database creation can be more comprehensive and manageable.

\subsection{VANET localization using relative distances measurements}

This approach takes advantage of the emerging VANET environments. The distances between VANET nodes are estimated and exchanged among vehicles along with preliminary estimates of the vehicles' locations. Vehicles can then use this information to construct local relative position maps that contain the vehicles and their neighbours. This strategy has initially emerged in Wireless Sensor Networks (WSN), but recently, a number of solutions have been proposed for use in VANET Benslimane (2005); Drawil \& Basir (2008); Parker \& Valaee (2007)

\subsubsection{Vehicle localization in VANET}

A VANET based localization method was introduced in Benslimane (2005) for localizing vehicles with no GPS receivers, or those whose location can not be determined because satellite signals have been lost, for instance, in a tunnel. With this method, vehicles that are not equipped with GPS determine their own locations by relying on information they receive from vehicles that are equipped with GPS. Vehicles within transmission range can measure the distances between each other using one of the radio-location methods presented in Caffery \& Stuber (1998). By finding its closest three neighbours the unequipped vehicle can compute its position using trilateration.

\subsubsection{Cooperative vehicle position estimation}

The work reported in Parker \& Valaee (2006) presents a method of distributed vehicle localization in VANET. The method utilizes RSS measurements to estimate the distances between one vehicle and others in its coverage area. It is assumed that vehicles initially estimate their own locations using a GPS receiver and then exchange their location information so that they can perform an optimization technique in order to improve their location estimates.

This technique demonstrates robustness of location estimates. However, it lacks the ability to detect and avoid the effect of multipath signals in the GPS measurements, which drastically degrades the localization accuracy in multipath environments (e.g., urban canyons).

In Drawil \& Basir (2010) an algorithm called InterVehicle-Communication-Assisted Localization (IVCAL) is proposed to mitigate the multipath effect in the location estimates of vehicles in VANET. A KF and an inter-vehicle-communication system collaborate in order to increase the robustness and accuracy of the localization of every vehicle in the network. The two main components that allow the inter-vehicle-communication system and the KF to interact are the Multipath Detection Unit (MDU), which detects the existence of a multipath effect in the output of the KF, and the Localization Enhancement Unit (LEU), which obtains the neighbours' information from the inter-vehicle-communication system and feeds an optimized location estimate back to the KF (Figure 4). As in Jabbour, Cherfaoui \& Bonnifait (2006) and Jabbour, Bonnifait \& Cherfaoui (2006), KF innovation is used as an indication of the contamination of the GPS measurement, and it has therefore been used as a learning pattern for the MDU in IVCAL. An uncertainty measure is also utilized in order to specify a subset of the most accurate network neighbours that can be used as anchors to enable vehicles to improve their location estimates. 
Lack of adequate location anchors and/or prolonged multipath conditions remain unsolved problems that continue to degrade localization accuracy.

\section{Multi-level fusion approaches}

As it has been reported above, a verity of multi-modality localization methods have evolved in recent years. Typical modalities include satellite signals, VANET communication, vision features, laser rays, etc. This variety of information has motivated the concept of multi-level fusion.

For instance, in Boukerche et al. (2008), a data-fusion model is proposed in the form of a three-level fusion localization system. In the first level, a variety of location information is gathered as row data and processed separately using local filters that are suitable for each type of location information. As with the system in Skog \& Handel (2009), the second level combines the output of the first level and produces a better location estimate. In Boukerche et al. (2008), the results are then fused in the third level based on contextual information (e.g., digital maps and traffic information). In this scheme, the final location estimate is fed back to the second level in order to improve future estimations.

Multi Level fusions aims to tackle data fusion as a hierarchical process so as to allow for combining measurements at various levels of abstraction in a simple manner. Nevertheless, if the estimates in the lowest-level filters are evaluated for reliability, the fusion of these estimates in higher-level filters will then be more robust.

\section{Integrity of localization systems}

Due to the inherent errors in the positioning information, a level of uncertainty in location estimates is inevitable. Therefore, it is essential to measure the reliability of the positioning information in order to identify any hidden anomalies. To achieve this task, a level of trust, integrity, in every estimate must be determined.

In the last two decades, a significant effort has been made in aviation to develop integrity monitoring systems Hewitson (2003); Walter \& Enge (1995). Integrity is defined as a measure

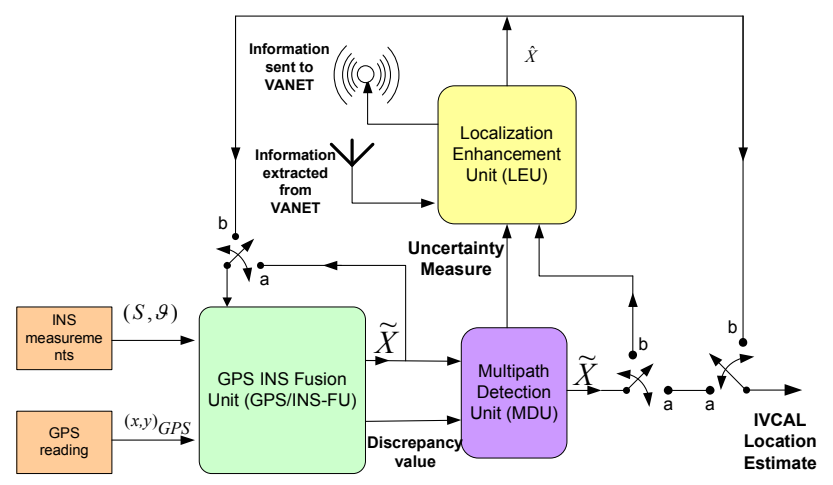

Fig. 4. Block diagram of the inter-vehicle-communication-assisted localization technique. 
of the trust which can be placed in the correctness of the information supplied by the total system; integrity includes the ability of a system to provide timely and valid measurements to users ESA (n.d.). Three key components have been proposed for integrity monitoring: 1) fault detection, 2) fault isolation, and 3) removal of faulty measurement sources from the estimates Hewitson et al. (2004). The European Geostationary Navigation Overlay Service (EGNOS) and the Wide Area Augmentation System (WAAS), Hewitson (2003), are developed to form a redundant source of information for the Global Navigation Satellite Systems (GNSS) in order to perform integrity monitoring by providing correction information.

During the last decade, monitoring the integrity of land-vehicles' localization has attracted attention due to the increasing demand for highly reliable accurate location data. Since roving in dense urban environments may limit access to the signals from augmentation systems such as EGNOS or WAAS, other means of measuring integrity have been proposed Schlingelhof et al. (2008).

For instance, Toledo-Moreo et al. (2006) presents a localization solution based on the fusion of GNSS and INS sensors. In this fusion process an interactive multimodel method is used. Different covariance matrices are used as a response to change in the noise behaviour. The proposed integrity measure is based on the covariance matrix of the EKF estimation error.

Relying on the error covariance matrix can be misleading especially when experiencing unmodeled environment noise. In other words, it is not possible in many cases to detect, isolate, and remove the estimation faults, let alone the unavoidable false alarms.

Also, in Jabbour et al. (2008) a binary integrity decision-maker is proposed for a map-matching localization technique in which multihypothesis road-tracking method combines proprioceptive sensors (odometers and gyrometers) with GPS and map information. In this work, the integrity represents high or low confidence of the location estimate. The candidate tracks or roads are associated with a probability that is computed using the multihypothesis road-tracking method. If one credible road exists and the normalized innovation is below a prespecified threshold, the technique declares high confidence location estimate. However, the lack of granularity in the integrity measure limits the range of the integrity-level based application that can use this method.

Integrity monitoring of map-matching localization has also been proposed and tested in Quddus (2006). However, in this work three indicators has been monitored to achieve this task: distance residuals, heading residuals, and an indicator related to uncertainty of the map matched position. Due to the linguistic nature of these indicators, they have been combined using a fuzzy inference model to produce a value between 0 to 100 to indicate the integrity of the system. The integrity threshold has been determined experimentally to be 70 , where the type of the environment experienced during the experiment was not specified. The value of the threshold thus can be considered specific to the environment of the experiment. Therefore, the approach might not guarantee a robust integrity monitoring. In other words, it is possible to come across an environment that influences the system to produce both an integrity value above the threshold and a location estimate mismatch.

\section{Performance criteria and benchmarking}

From the discussion above it is clear that vehicle localization is an increasingly growing area of research. Nevertheless, there is a number of outstanding issues that still need to be addressed. 
In order to put these outstanding issues in practical context the following performance criteria are proposed.

7.1. Accuracy: Accuracy of a vehicle location estimate is defined as the degree of closeness of a vehicle's location estimate to its actual (true) location.

7.2. Availability: Availability of a vehicle location estimate is defined as the ratio of the number of estimates produced to the number of estimates expected per one unit of time.

7.3. Response Time: Response time is the time required by a localization technique to produce a location estimate.

7.4. Integrity: Integrity is defined as the level of confidence that can be placed in the correctness of the location estimate Bakhache \& Nikiforov (2000); ESA (n.d.); Quddus (2006).

Based on the above performance criteria, a benchmark can be established in order to compare the performance of different localization techniques based on reported best achievable accuracy localization performance. Localization performance is compared with respect to reliability as well. Table 1 provides a summary of the comparison in terms of modality used, best case accuracy, environmental constraints, synchronization requirements, and dependency on infrastructure. Table 2 reports emerging applications and their requirements

\begin{tabular}{|c|c|c|c|c|}
\hline Modality(ies) & Best Case Accuracy (m) & Availability & \multicolumn{2}{|c|}{ Synch. Infr.str. } \\
\hline GPS & 10-20 Hoshen (1996); Leva (1996) & Out Door-Open Sky & Yes & No \\
\hline DeadReckoning (DR) & Worsen with time Kao (1991) & Anywhere & No & No \\
\hline DGPS with Visible Satellites & 0.01-7.6 Meguro et al. (2009) & suburban-Open Sky & Yes & Yes \\
\hline DGPS+DR+Map Matching & 0.5-5 Lahrech et al. (2005) & Out Door-Open Sky & $\mathrm{N} / \mathrm{A}$ & Yes \\
\hline GPS+Vision+Map Matching & 0.5-1 Chausse et al. (2005); Jabbour, Bonnifait \& Cherfaoui (2006) & Out Door-Open Sky & No & Yes \\
\hline Cellular Localization & 90-250 Chen et al. (2006); 25-69 Porretta et al. (2008) & Under Network Coverage & Yes & Yes \\
\hline Location Services & Submeter Zhang et al. (2008) & In Door & $\mathrm{N} / \mathrm{A}$ & Yes \\
\hline Relative Ad hoc Localization & 2-7 (Simulation Drawil \& Basir (2008); Parker \& Valaee (2006)) & Suburban & Yes & No \\
\hline
\end{tabular}

Table 1. Specifications of Localization Techniques.

with respect to localization accuracy. It is evident from Tables 1 and 2 that current localization techniques do not live up to the required integrity and availability performance. In other words, the delivered performance of the localization techniques listed in Table 1 is not always above the target performance specified by the applications, and that is due to the unavailability of their measurements or the decrease in their accuracy in some environments, such as urban canyons, foggy weather, and dark areas.

Hence, performance needed by applications can constitute a challenging issue in the fusion process of a multi-sensory system. Therefore task driven integrity issues relevant to vehicle localization are highlighted next.

\section{Task driven localization integrity}

From the above discussion it is obvious that for localization systems to meet the expectations of emerging applications it is imperative that they employ diverse location measurement sources and effective strategies to fuse these sources so as to achieve the Quality of Service expected of them. Of course this Quality of Service is multi-dimensional as it pertains to expected accuracy, availability, response time and integrity. The Quality of Service as a function of these performance criteria is application and task dependent. The more stringent is the required Quality of Service with respect to a given performance criterion, the more resources are needed and the higher is the computational cost. This presents a challenge for 


\begin{tabular}{lcc}
\hline \multirow{2}{*}{ Application } & \multicolumn{2}{c}{ Required Accuracy } \\
\cline { 2 - 3 } & Low(10-20 m) & Medium (1-5 m) High (less than 1 m) \\
\hline Message Routing (VANET) & $\mathrm{X}$ & \\
Data Dissemination & $\mathrm{X}$ & \\
Map Localization & $\mathrm{X}$ & $\mathrm{X}$ \\
Coop. Cruise Control & & $\mathrm{X}$ \\
Coop. Intersection Safety & $\mathrm{X}$ & \\
Blind Crossing & $\mathrm{X}$ & $\mathrm{X}$ \\
Platooning & & $\mathrm{X}$ \\
Collision Warning Sys. & & $\mathrm{X}$ \\
Vision Enhancement & & $\mathrm{X}$ \\
Automatic Parking & & \\
Road Pricing & & \\
\hline
\end{tabular}

Table 2. Applications Requirement for Location Estimates Boukerche et al. (2008).

the system as calls for effective use of resources to achieve the target Quality of Service. For example, there are applications where accuracy can be traded for faster response time. On the other hand, there are applications where response time is not as important as accuracy (offline vehicle track mapping). There are also applications where both requirements, accuracy and response time, can not be compromised for any other gain.

Indeed, task or goal driven localization is about effective allocating system resources and planning of localization tasks such that the system mission is achieved with maximum integrity possible. This strategy to performance is a key issue to the new trends of hybrid localization systems. In order for this strategy to work it is imperative that the impact of the environment is not ignored. Without modeling the impact of the environment on the system, the system can not be guaranteed to achieve its target performance, and even worst as it may falsely determine its task is accomplished. Thus, modeling the impact of the environmental conditions on the system is a central issue to the following proposed framework.

\section{Task-driven localization through integrity assessment and control}

It is well understood that the reported techniques can estimate the location of vehicles relatively accurately in some situations if they are given adequate time to perform the task. However, they may not perform as well in other situations. The deficiencies of these localization techniques are uncorrelated as they are expected to be of diverse phenomena, and/or utilize different algorithmic paradigms. This motivates the development of systems that can take advantage of this diversity to achieve a reliable and accurate performance.

In this section, a high level concept of a novel framework for fusing different localization techniques is proposed, Figure 5. What distinguishes this framework from existing ones is its ability to take in account the impact of the measurement conditions on the individual techniques. Thus, it is able to optimize the fusion process so as to maximize the accuracy and integrity of the localization estimates. The framework consists of three logical layers: (1) Primary Localization layer which provides preliminary location estimates using the available localization techniques; (2) Integrity Monitoring layer which computes the reliability of the vehicle's location estimates produced by the Primary Localization layer- a process that captures the impact of measurement conditions; and (3) Estimate Fusion and Management layer which interacts with the application task to ensure that the task's expected localization 


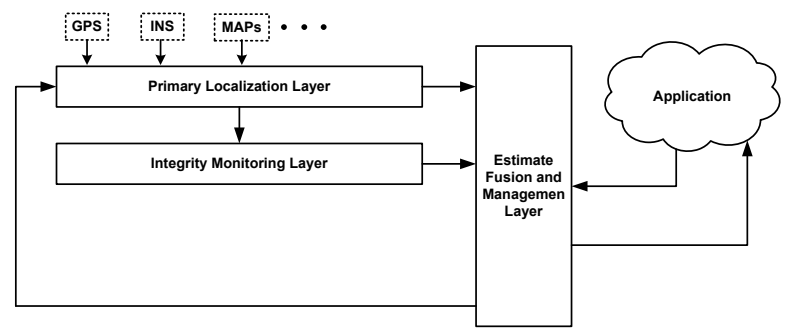

Fig. 5. The structure of the proposed framework.

accuracy and integrity are achieved by executing a proper fusion scheme. In what follows a further description of the framework layers functionality.

\subsection{Primary localization layer}

The primary localization layer comprises of the system's localization techniques which are partitioned in the form of a set of Primary Localization Units (PLUs), as can be seen in Figure 6. Any localization technique, such as those mentioned above, can be used in any given PLU. These primary localization units receive localization requests from the Estimate Fusion and Management layer. Each PLU is constructed from techniques that are based on different phenomena/algorithms to ensure minimum correlation. A primary localization unit can share its information sources with other units; it can constitute a single modality or multiple-modalities. An example of a single modality PLU is one that estimates the vehicle

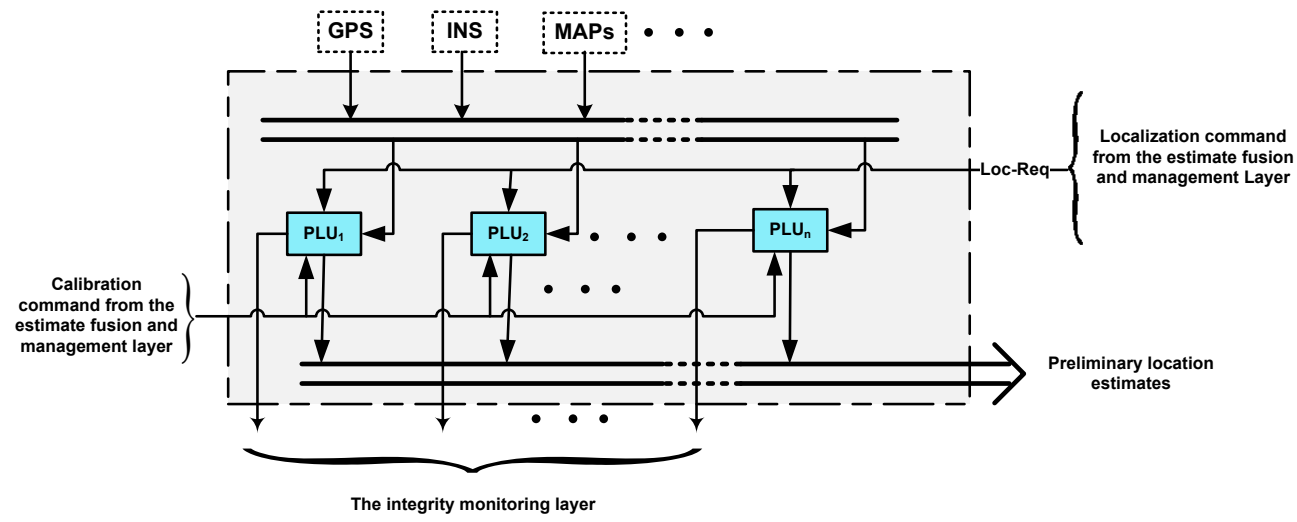

Fig. 6. Primary localization layer.

location from a GPS information source. IVCAL is an example of a PLU that utilizes three modalities: GPS, INS, and Inter-Vehicle-Communication.

\subsection{Integrity Monitoring layer}

Central to the proposed framework is the integrity monitoring layer. Here, an Integrity Monitoring unit (IMU) is used to monitor the performance of a primary localization unit (Figure 7). The monitoring process takes in consideration the impact of the measurement conditions on the PLU. For example, to indicate the reliability of an estimate DOP measure 


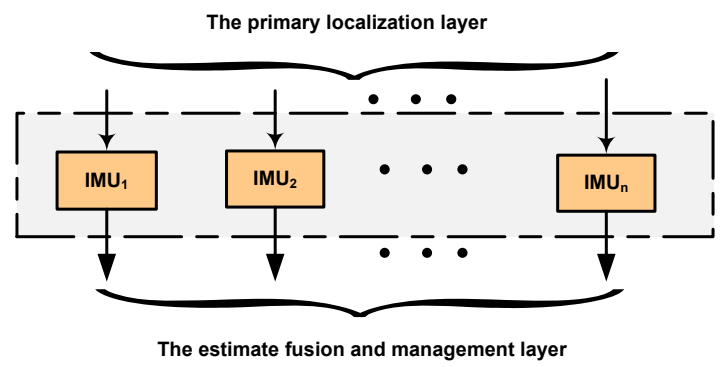

Fig. 7. Integrity monitoring layer.

and/or the signal to noise ratio can be utilized when a GPS receiver is used, light intensity can be utilized when vision features are used, and KF innovation can be utilized when IVCAL is used. Various tools can be employed in this layer based on the type of the localization technique. Fuzzy inference systems and probabilistic models for reliability are two examples of these tools.

\subsection{The Estimate Fusion and Management Layer}

The Estimate Fusion and Management layer (EFM) is responsible for determining an effective integration (Meta-Fusion) strategy for fusing the estimates produced by the different primary localization units so as to achieve the required localization accuracy and integrity (Figure 8). The estimate fusion and management processes the location estimates produced by the

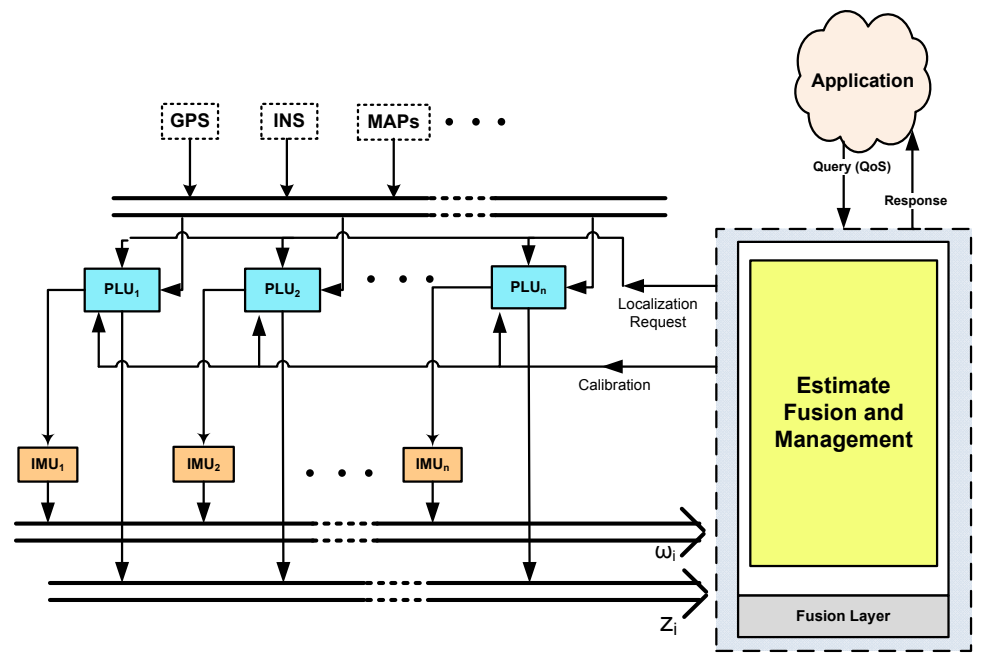

Fig. 8. Estimate fusion and management layer.

different primary localization units in conjunction with their integrity assessments. Since the vehicle is expected to be performing localization while moving, it is imperative for the fusion process to perform spatial and temporal alignment of the estimates produced by the different PLUs. Therefore, this layer employs a synchronization handler to manage timing issues among the different PLUs. Given the task's target accuracy and integrity, as well as 
that of the various PLUs, a management and fusion scheme is computed such that the scheme produces a location estimate that meets the task requirements.

To overcome the problems of this layer, first of all, PLU estimates should be time-stamped as close as possible to a common time base. Of course the allowable synchronization error would depend on factors such as the speed of the vehicle relative to the PLU response time. It is also affected by the system's desired spatial precision and detection frequency. The tighter time synchronization is achieved with respect to the common time base, the greater precision is possible in the tracking of the vehicle.

Second of all, since the fusion process is task driven, an optimal fusion strategy is the one that achieves the target accuracy and integrity within the constraints of the task deadline. This gives rise to the challenge of optimal estimate fusion and reliability aggregation. Both fuzzy reasoning and evidential reasoning are a tentative tools to be investigated as the bases for constructing the meta-fusion model. Fuzzy reasoning can be used for representing uncertainty in the estimates as well as for representing linguistic task requirements. Since some PLUs may employ probabilistic (Bayesian) estimators, it will be interesting to study how probabilistic estimates and fuzzy estimates are represented in a unified uncertainty framework.

Bayesian theory based fusion techniques have been evolving in fields such as process control, target tracking and object recognition. Nonetheless, effective fusion performance can only be achieved if adequate and appropriate priori and conditional probabilities are available. Although, at least in some situations, assumptions can be made with respect to priori and posteriori probabilities, these assumptions can turn to be unreasonable in many other situations, especially if we are to allow for non-probabilistic estimators in the PLU layer. One possible solution is using the Dempster-Shafer (DS) evidence theory as an extension to the Bayes theory. DS belief and plausibility functions can be used to quantify evidence and unify uncertainty of the PLU estimates. DS evidence theory can also model how the uncertainty of a given location estimate diminishes as pieces of evidence accumulate during the localization process. One important aspect of this theory is that reasoning or decision making can be carried out with incomplete or conflicting pieces of evidence - a reality that is quit common in localization problems.

\section{Conclusions}

In this chapter, a variety of reported localization techniques are presented and classified based on the type of the measurement of the location information used.

Although, techniques that incorporate fusion of motion sensory data with GPS localization have demonstrated improvement in performance, there are still situations that can have a negative effect on their localization accuracy. Incremental localization errors in motion-sensor data and the multipath effect in urban canyon environments contribute significantly to such location estimate errors, which necessitates augmenting the initial location data with other sources of location information in order to overcome these shortcomings.

Digital maps and visual features enhance GPS-DR localization by recognizing landmarks in the surrounding environment and matching them with others in a reference GIS map. A key problem associated with this scheme is that the landmark segmentation process is complex and ill conditioned process.

Multi-level fusion schemes are promising as they employ multiple location measurement phenomena. However, these schemes have given birth to new challenges in the localization 
problem in terms of resource synchronization, resource management, and task driven performance.

A novel framework for vehicle localization is presented. The aim is to develop a vehicle localization system that can optimize and plan the use of its resources so as to achieve the performance requirements of the localization task or application. The main components of the proposed framework are key research issues.

\section{References}

Al-Bayari, O. \& Sadoun, B. (2005). New Centralized Automatic Vehicle Location Communications Software System Under GIS Environment, INTERNATIONAL JOURNAL OF COMMUNICATION SYSTEMS 18(9): 833.

Al-Jazzar, S. \& Caffery, J., J. (2004). NLOS Mitigation Method for Urban Environments, IEEE 60th Vehicular Technology Conference 7: 5112-5115.

Aono, T., Fujii, K., Hatsumoto, S. \& Kamiya, T. (1998). Positioning of Vehicle on Undulating Ground using GPS and Dead Reckoning, IEEE International Conference on Robotics and Automation 4: 3443-3448.

Bakhache, B. \& Nikiforov, I. (2000). Reliable detection of faults in measurement systems, International Journal of Adaptive Control and Signal Processing 14(7): 683-700.

Benslimane, A. (2005). Localization in Vehicular Ad hoc Networks, Systems Communications. Proceedings pp. 19-25.

Bouju, A., Stockus, A., Bertrand, R. \& Boursier, P. (2002). Location-Based Spatial Data Management in Navigation Systems, IEEE Intelligent Vehicle Symposium 1: 172-177.

Boukerche, A., Oliveira, H., Nakamura, E. \& Loureiro, A. (2008). Vehicular Ad hoc Networks: A New Challenge for Localization-Based Systems, Computer Communications 31(12): 2838-2849.

Bouvet, D. \& Garcia, G. (2000). Improving the Accuracy of Dynamic Localization Systems using RTK GPS by Identifying the GPS Latency, IEEE International Conference on Robotics and Automation 3: 2525-2530 vol.3.

Caffery, J. (1999). Wireless Location in Cdma Cellular Radio Systems, Kluwer Academic Pub.

Caffery, J. \& Stuber, G. (1994). Vehicle Location and Tracking for IVHS in CDMA Microcells, 5th IEEE International Symposium on Personal, Indoor and Mobile Radio Communications 4: 1227-1231.

Caffery, J. \& Stuber, G. (1998). Overview of Radiolocation in CDMA Cellular Systems, IEEE Communications Magazine 36(4): 38 - 45.

Caffery, J.J., J. (2000). A New Approach to the Geometry of TOA Location, IEEE 52nd Vehicular Technology Conference 4: 1943-1949.

Chausse, F., Laneurit, J. \& Chapuis, R. (2005). Vehicle Localization on a Digital Map using Particles Fltering, pp. 243-248.

Chen, M., Sohn, T., Chmelev, D., Haehnel, D., Hightower, J., Hughes, J., LaMarca, A., Potter, F., Smith, I. \& Varshavsky, A. (2006). Practical Metropolitan-scale Positioning for GSM Phones, Lecture Notes in Computer Science 4206: 225.

Cramer, M. (1997). GPS/INS Integration.

Dao, D., Rizos, C. \& Wang, J. (2002). Location-Based Services: Technical and Business Issues, GPS Solutions 6(3): 169-178.

Drawil, N. \& Basir, O. (2008). Vehicular Collaborative Technique for Location Estimate Correction, IEEE 68th Vehicular Technology Conference pp. 1-5. 
Drawil, N. \& Basir, O. (2010). Intervehicle-communication-assisted localization, Intelligent Transportation Systems, IEEE Transactions on 11(3): 678 -691.

ESA (n.d.). Making EGNOS Work for You,CD-ROM.

Fuerstenberg, K. \& Weiss, T. (2005). Feature-Level Map Building and Object Recognition for Intersection Safety Applications, Proceedings of IEEE Intelligent Vehicles Symposium pp. 490-495.

Hewitson, S. (2003). GNSS receiver autonomous integrity monitoring: A separability analysis, Proc. ION GPS pp. 1502-1509.

Hewitson, S., Kyu Lee, H. \& Wang, J. (2004). Localizability Analysis for GPS/Galileo Receiver Autonomous Integrity Monitoring, The Journal of Navigation 57(02): 245-259.

Honghui, Q. \& Moore, J. B. (2002). Direct Kalman Filtering Approach for GPS/INS Integration, IEEE Transactions on Aerospace and Electronic Systems 38(2): 687 - 693.

Hoshen, J. (1996). The GPS Equations and the Problem of Apollonius, Aerospace and Electronic Systems, IEEE Transactions 32(3): 1116 - 1124.

Jabbour, M., Bonnifait, P. \& Cherfaoui, V. (2006). Enhanced Local Maps in a GIS for a Precise Localisation in Urban Areas, IEEE Intelligent Transportation Systems Conference pp. 468-473.

Jabbour, M., Bonnifait, P. \& Cherfaoui, V. (2008). Map-Matching Integrity using Multi-Sensor Fusion and Multi-Hypothesis Road Tracking, Journal of Intelligent Transportation Systems Technology Planning and Operations 12(4): 189-201.

Jabbour, M., Cherfaoui, V. \& Bonnifait, P. (2006). Management of Landmarks in a GIS for an Enhanced Localisation in Urban Areas, Intelligent Vehicles Symposium, 2006 IEEE pp. 50-57.

Kao, W. (1991). Integration of GPS and Dead-Reckoning Navigation Systems, Vehicle Navigation and Information Systems Conference, 1991 2: 635-643.

Lahrech, A., Boucher, C. \& Noyer, J.-C. (2005). Accurate Vehicle Positioning in Urban Areas, 31st Annual Conference of IEEE Industrial Electronics Society p. 5 pp.

Lai, C.-C. \& Tsai, W.-H. (2003). Location Estimation and Trajectory Prediction of Moving Lateral Vehicle using Two Wheel Shapes Information in 2-D Lateral Vehicle Images by 3-D Computer Vision Techniques, IEEE International Conference on Robotics and Automation 1: 881-886.

Le, B. L., Ahmed, K. \& Tsuji, H. (2003). Mobile Location Estimator With NLOS Mitigation using Kalman Filtering, Wireless Communications and Networking, IEEE 3: 1969-1973.

Leva, J. L. (1996). An Alternative Closed-Form Solution to the GPS Pseudo-Range Equations, Aerospace and Electronic Systems, IEEE Transactions 32(4): 1430 - 1439.

McGuire, M., Plataniotis, K. \& Venetsanopoulos, A. (2003). Location of Mobile Terminals using time Measurements and Survey Points, IEEE Transactions on Vehicular Technology Conference 52(4): 999-1011.

Meguro, J.-i., Murata, T., Takiguchi, J.-i., Amano, Y. \& Hashizume, T. (2009). GPS Multipath Mitigation for Urban Area Using Omnidirectional Infrared Camera, IEEE Transactions on Intelligent Transportation Systems 10(1): 22-30.

Nishimura, Y., Tanahashi, I., Taniguchi, S., Matsumoto, N. \& Nakamura, K. (1996). A New Concept for Vehicle Localization of Road Debiting System, Proceedings of the IEEE Intelligent Vehicles Symposium pp. 93-98.

Parker, R. \& Valaee, S. (2006). Vehicle Localization in Vehicular Networks, IEEE 64th Vehicular Technology Conference pp. 1-5.

Parker, R. \& Valaee, S. (2007). Cooperative Vehicle Position Estimation, IEEE International Conference on Communications pp. 5837-5842. 
Porretta, M., Nepa, P., Manara, G. \& Giannetti, F. (2008). Location, Location, Location, Vehicular Technology Magazine, IEEE 3(2): 20-29.

Quddus, M. (2006). High integrity map matching algorithms for advanced transport telematics applications, PhD thesis, Citeseer.

Rae, A. \& Basir, O. (2007). A Framework for Visual Position Estimation for Motor Vehicles, 4th Workshop on Positioning, Navigation and Communication pp. 223-228.

Rezaei, S. \& Sengupta, R. (2005). Kalman Filter Based Integration of DGPS and Vehicle Sensors for Localization, Mechatronics and Automation, IEEE International Conference 1: 455-460.

Sayed, A., Tarighat, A. \& Khajehnouri, N. (2005). Network-Based Wireless Location: Challenges Faced in Developing Techniques for Accurate Wireless Location Information, Signal Processing Magazine, IEEE 22(4): 24-40.

Schlingelhof, M., Betaille, D., Bonnifait, P. \& Demaseure, K. (2008). Advanced Positioning Technologies for Co-operative Systems, Intelligent Transport Systems, IET 2(2): 81-91.

Sharaf, R., Noureldin, A., Osman, A. \& El-Sheimy, N. (2005). Online INS/GPS Integration with A Radial Basis Function Neural Network, IEEE Aerospace and Electronic Systems Magazine 20(3): 8-14.

Skog, I. \& Handel, P. (2009). In-Car Positioning and Navigation TechnologiesâĂŤA Survey, IEEE Transactions on Intelligent Transportation Systems 10(1): 4-21.

Sliety, M. (2007). Impact of Vehicle Platform on Global Positioning System Performance in Intelligent Transportation, Intelligent Transport Systems, IET 1(4): 241-248.

Smith, W.W., J. (1991). Passive Location of Mobile Cellular Telephone Terminals, IEEE International Carnahan Conference on Security Technology pp. 221-225.

Stockus, A., Bouju, A., Bertrand, F. \& Boursier, P. (2000). Web-Based Vehicle Localization, Proceedings of the IEEE Intelligent Vehicles Symposium pp. 436-441.

Toledo-Moreo, R., Zamora-Izquierdo, M. \& Gomez-Skarmeta, A. (2006). A Novel Design of a High Integrity Low Cost Navigation Unit for Road Vehicle Applications, pp. 577-582.

Venkatraman, S., Caffery, J., J. \& You, H.-R. (2002). Location using LOS Range Estimation in NLOS Environments, Vehicular Technology Conference, IEEE 55th 2: 856-860.

Walter, T. \& Enge, P. (1995). Weighted RAIM for precision approach, PROCEEDINGS OF ION GPS, Vol. 8, Citeseer, pp. 1995-2004.

Wang, X., Wang, Z. \& O'Dea, B. (2003). A TOA-Based Location Algorithm Reducing the Errors due to Non-Line-of-Sight (NLOS) Propagation, IEEE Transactions on Vehicular Technology Conference 52(1): 112-116.

Weiss, T., Kaempchen, N. \& Dietmayer, K. (2005). Precise ego-Localization in Urban Areas using Laserscanner and High Accuracy Feature Maps, Proceedings of IEEE Intelligent Vehicles Symposium pp. 284-289.

Wylie, M. \& Holtzman, J. (1996). The Non-Line of Sight Problem in Mobile Location Estimation, 5th IEEE International Conference on Universal Personal Communications 2: 827-831.

Zhang, G., Krishnan, S., Chin, F. \& Ko, C. C. (2008). UWB Multicell Indoor Localization Experiment System with Adaptive TDOA Combination, pp. 1-5. 


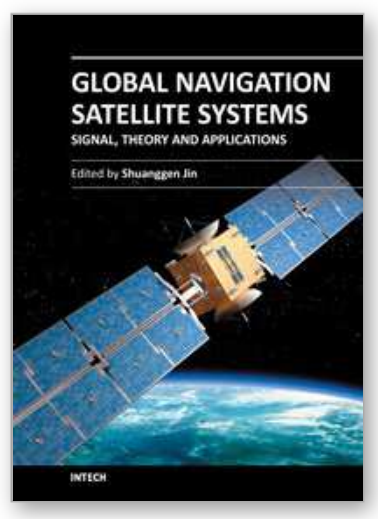

\author{
Global Navigation Satellite Systems: Signal, Theory and \\ Applications \\ Edited by Prof. Shuanggen Jin
}

ISBN 978-953-307-843-4

Hard cover, 426 pages

Publisher InTech

Published online 03, February, 2012

Published in print edition February, 2012

Global Navigation Satellite System (GNSS) plays a key role in high precision navigation, positioning, timing, and scientific questions related to precise positioning. This is a highly precise, continuous, all-weather, and real-time technique. The book is devoted to presenting recent results and developments in GNSS theory, system, signal, receiver, method, and errors sources, such as multipath effects and atmospheric delays. Furthermore, varied GNSS applications are demonstrated and evaluated in hybrid positioning, multi-sensor integration, height system, Network Real Time Kinematic (NRTK), wheeled robots, and status and engineering surveying. This book provides a good reference for GNSS designers, engineers, and scientists, as well as the user market.

\title{
How to reference
}

In order to correctly reference this scholarly work, feel free to copy and paste the following:

Nabil Drawil and Otman Basir (2012). Emerging New Trends in Hybrid Vehicle Localization Systems, Global Navigation Satellite Systems: Signal, Theory and Applications, Prof. Shuanggen Jin (Ed.), ISBN: 978-953-307843-4, InTech, Available from: http://www.intechopen.com/books/global-navigation-satellite-systems-signaltheory-and-applications/emerging-new-trends-in-hybrid-vehicle-localization-systems

\section{INTECH}

open science | open minds

\section{InTech Europe}

University Campus STeP Ri

Slavka Krautzeka 83/A

51000 Rijeka, Croatia

Phone: +385 (51) 770447

Fax: +385 (51) 686166

www.intechopen.com

\section{InTech China}

Unit 405, Office Block, Hotel Equatorial Shanghai

No.65, Yan An Road (West), Shanghai, 200040, China

中国上海市延安西路65号上海国际贵都大饭店办公楼405单元

Phone: +86-21-62489820

Fax: +86-21-62489821 
(C) 2012 The Author(s). Licensee IntechOpen. This is an open access article distributed under the terms of the Creative Commons Attribution 3.0 License, which permits unrestricted use, distribution, and reproduction in any medium, provided the original work is properly cited. 